\title{
Strain mapping at propagating interfaces in pseudoelastic NiTi
}

\author{
A. Schaefer ${ }^{\mathrm{a}, 1}$ and M. F.-X. Wagner ${ }^{1}$ \\ Ruhr-Universität Bochum, 44780 Bochum, Germany \\ ${ }^{1}$ Institute of Materials, Ruhr-Universität Bochum, 44780 Bochum
}

\begin{abstract}
Pseudoelastic NiTi often exhibits an inhomogeneous martensitic phase transformation: Martensite bands propagate through the specimen, distinct phase interfaces separate transformed from untransformed regions, and local strains increase step-like but smoothly by $\sim 5 \%$ across these interfaces. In the present study, we use an advanced strain mapping method (digital image correlation) to characterize local strain states and strain rates at propagating phase interfaces in a thin NiTi ribbon. The resulting strain data allows a detailed analysis of the interface geometry. The narrow transition regions are characterized by a finite width of $4-5 \mathrm{~mm}$ which generally increases near the outer edges of the specimen. Furthermore, the maximum local strain rates at the interfaces are one order of magnitude higher than the macroscopic strain rate that is usually used to characterize thermo-mechanical loading conditions.
\end{abstract}

\section{Introduction}

The unique properties of NiTi (thermal and mechanical shape memory) are related to a reversible martensitic phase transformation between a high symmetry, high temperature phase (austenite) and a low symmetry, low temperature phase (martensite). Pseudoelastic deformation is associated with a stress-induced formation of martensite and with transformation strains of $\sim 6-8 \%$ that can be fully recovered during unloading. When interpreting experimental stress-strain data obtained by tensile testing of thin specimens, it is important to take into account that these stress-induced transformations often occur in a strongly localized manner. During loading, martensite bands nucleate due to a local stress-concentration (e.g., near the grips due to the additional clamping pressure or because of stress raisers like geometric imperfections and notches). Distinct martensite-austenite phase interfaces separate the fully martensitic transformation bands and the yet untransformed austenitic parts of the specimen.

While there is a large number of previous studies on the effect of localization of deformation on the mechanical behavior of NiTi [1-6], a detailed analysis of strains near the moving phase interfaces has not been reported so far. Such careful observations are clearly needed to further our understanding of interface propagation and to provide a sound basis for modeling studies. One experimental method that can provide strain data with high spatial and temporal resolution is the digital image correlation (DIC) technique. By optically tracking and correlating the displacements of random speckle patterns on a specimen's surface, local displacement fields and the corresponding surface strain fields can be determined (see also Fig. 2). In previous studies on NiTi, DIC was successfully utilized to characterize strain fields in polycrystalline NiTi specimens with geometric features like holes and notches [7], and in precipitated NiTi single crystals [8]. These studies show that DIC measurements allow for accurate strain measurements despite the large strains and strain gradients involved. In the present work, we use DIC for in-situ measurements of local displacements, strains, and strain rates across phase interfaces in pseudoelastic NiTi ribbon specimens subjected to uniaxial tensile tests. Special emphasis is placed on the vicinities of the interfaces, and the spatial distribution of strains is carefully analyzed. In addition, the relationship between the applied macroscopic displacement rate and the velocity of interface propagation is explored. Finally, it is demonstrated that the macroscopic displacement rate is a characteristic experimental parameter that accounts for both the experimental setup as well as for the thermo-mechanical loading conditions.

\footnotetext{
a e-mail: Andreas.Schaefererub.de
}

This is an Open Access article distributed under the terms of the Creative Commons Attribution-Noncommercial License (http://creativecommons.org/licenses/by-nc/3.0/), which permits unrestricted use, distribution, and reproduction in any noncommercial medium, provided the original work is properly cited. 


\section{Experimental}

NiTi ribbons (width $3.3 \mathrm{~mm}$, thickness $0.68 \mathrm{~mm}$ ) with a nominal composition of 50.9 at.-\% Ni were obtained from Memory Metalle (Weil am Rhein, Germany). The ultra fine grained material is pseudoelastic at room temperature. Further details on microstructure and transformation temperatures have been published elsewhere [9]. Uniaxial tensile tests (free length of the specimens: $1_{0}=35 \mathrm{~mm}$ ) were performed at $22^{\circ} \mathrm{C}$ using a MTS 858 Mini Bionix II servo hydraulic machine equipped with a $2.5 \mathrm{kN}$ load cell and at a displacement rate of $0.5 \mathrm{~mm} / \mathrm{min}$. The speckle pattern was created by carefully spraying black and white paint onto the surface of the specimen (see also Fig. 2b). The setup for DIC consisted of one pair of 5 megapixel cameras that acquired images in situ with a rate of $0.2 \mathrm{~Hz}$ during one pseudoelastic loading-unloading cycle. Strains and strain rates were determined from the displacement fields by use of the software Vic3D (Limess, Pforzheim, Germany).

\section{Results and Discussion}

The stress-strain hysteresis recorded during the DIC experiment is shown in Fig. 1. In this graph, the strain associated with each dot represents a DIC measurement of the macroscopic strain averaged over the free length of the specimen. Such strain values closely resemble those recorded by conventional clip-on extensometers, which also determine the average strain within the gauge length. In fact, the stress-strain data in Fig. 1 is in excellent agreement with conventional extensometer measurements performed on similar specimens during tensile straining, [10]. The NiTi ribbon exhibits typical pseudoelastic behavior with distinct stress plateaus that are associated with the forward and reverse transformations.

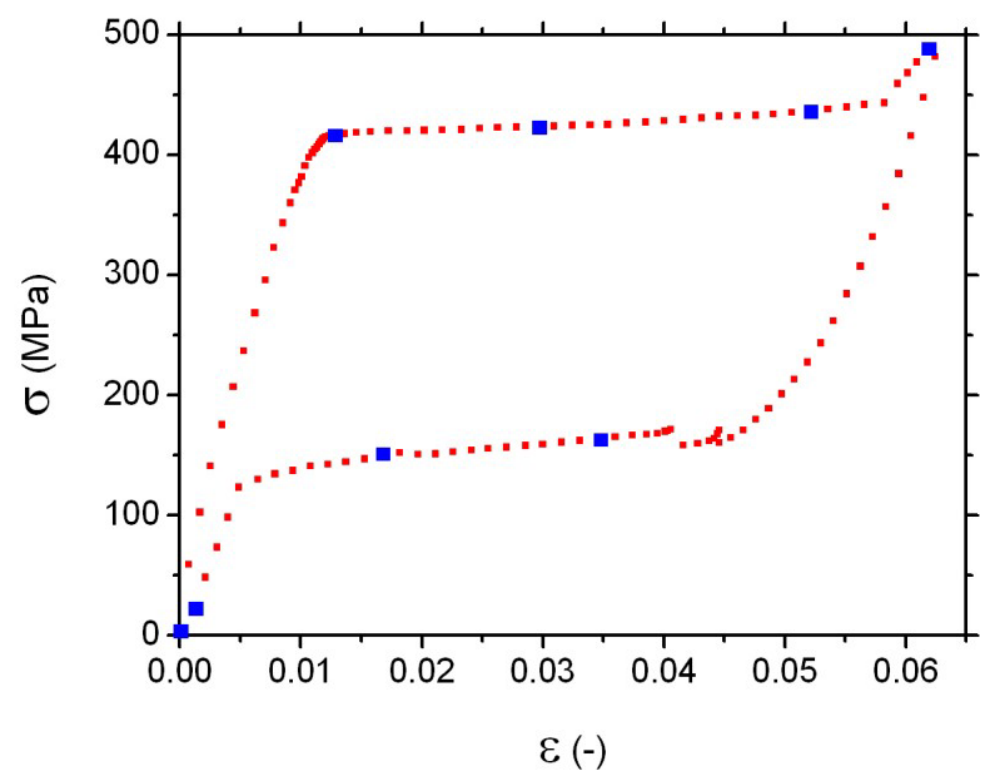

Fig. 1. Pseudoelastic stress-strain hysteresis of the NiTi ribbon. Strain values were determined from the DIC data as average strain of the specimen's free length. Each data point represents one DIC measurement. The eight blue dots correspond to the color scaled sequence in Fig. 2a).

A sequence of selected DIC images that represent the complete loading/unloading cycle is shown in Fig. 2a). The corresponding stress-strain points are also highlighted in Fig. 1. The color-coded DIC images represent the local strain distribution in the tensile direction, as computed from the displacements of the paint speckles on the specimen surface, Fig. 2b). A comparison of Fig. 1 and Fig. 2 clearly demonstrates that the onset of the pseudoelastic stress-plateau is associated with the nucleation of martensite bands near the grips of the experimental setup. These bands then propagate towards the middle of the specimen during further straining until they finally coalesce. Similarly, during unloading, austenitic bands are also nucleated near the grips and propagate towards the center of the specimen. During the elastic deformation of austenite and, beyond the pseudoelastic plateau, of martensite, the tensile strain is constant within the gauge length. More importantly, the tensile strains are also constant inside the austenitic/ martensitic regions during localized deformation, i.e., at stresses corresponding to the pseudoelastic plateau stress. The strain fields (with strains corresponding to the onsets and the ends of the pseudoelastic plateaus during loading and unloading and in the austenitic and martensitic regions, respectively) presented in Fig. 2a indicate that - while individual grains may well remain 
austenitic until higher stresses are applied, and additional deformation mechanisms such as detwinning or reorientation may also occur during further straining $[11,12]$ - the stress-induced martensitic transformation is (almost) complete inside the martensite bands from a macroscopic point of view. Therefore, at any given moment during pseudoelastic straining, additional transformation occurs exclusively in the transition regions between the fully martensitic and fully austenitic regions. These macroscopic phase interfaces have a finite width of several millimeters and exhibit a characteristic inclination with respect to the tensile axis.

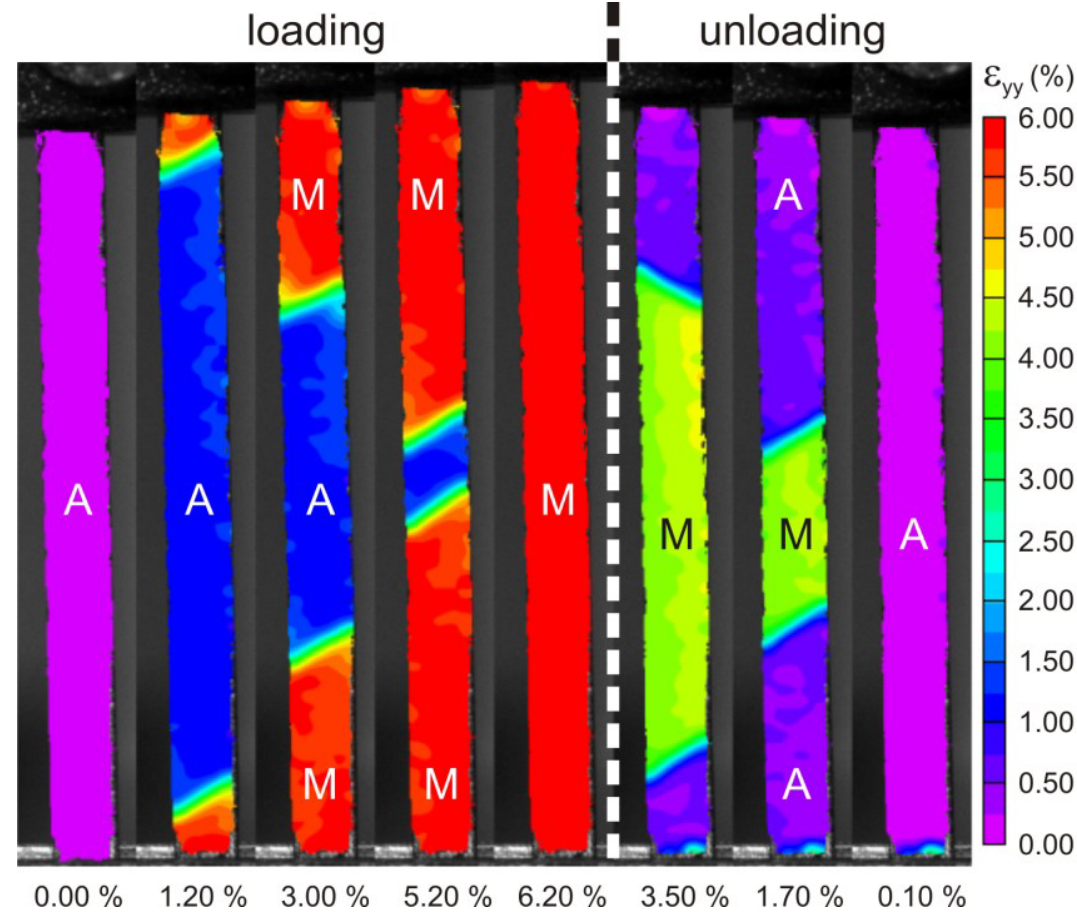

a)

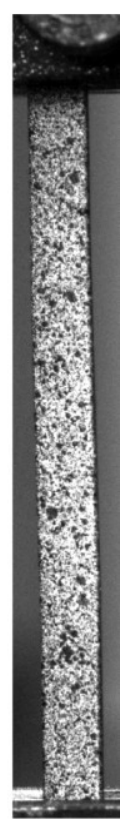

b)

Fig. 2. a) Sequence of selected DIC-measurements. Colors represent the local tensile strain (macroscopic strains are given below each strain map). " $\mathrm{A}$ " and " $\mathrm{M}$ " indicate austenitic or martensitic regions respectively. During loading, martensite bands form at the grips of the specimen and then propagate towards the middle of the specimen during further straining. When the specimen is unloaded, austenitic bands are also nucleated at the grips. b) Random speckle pattern on the surface of the specimen.

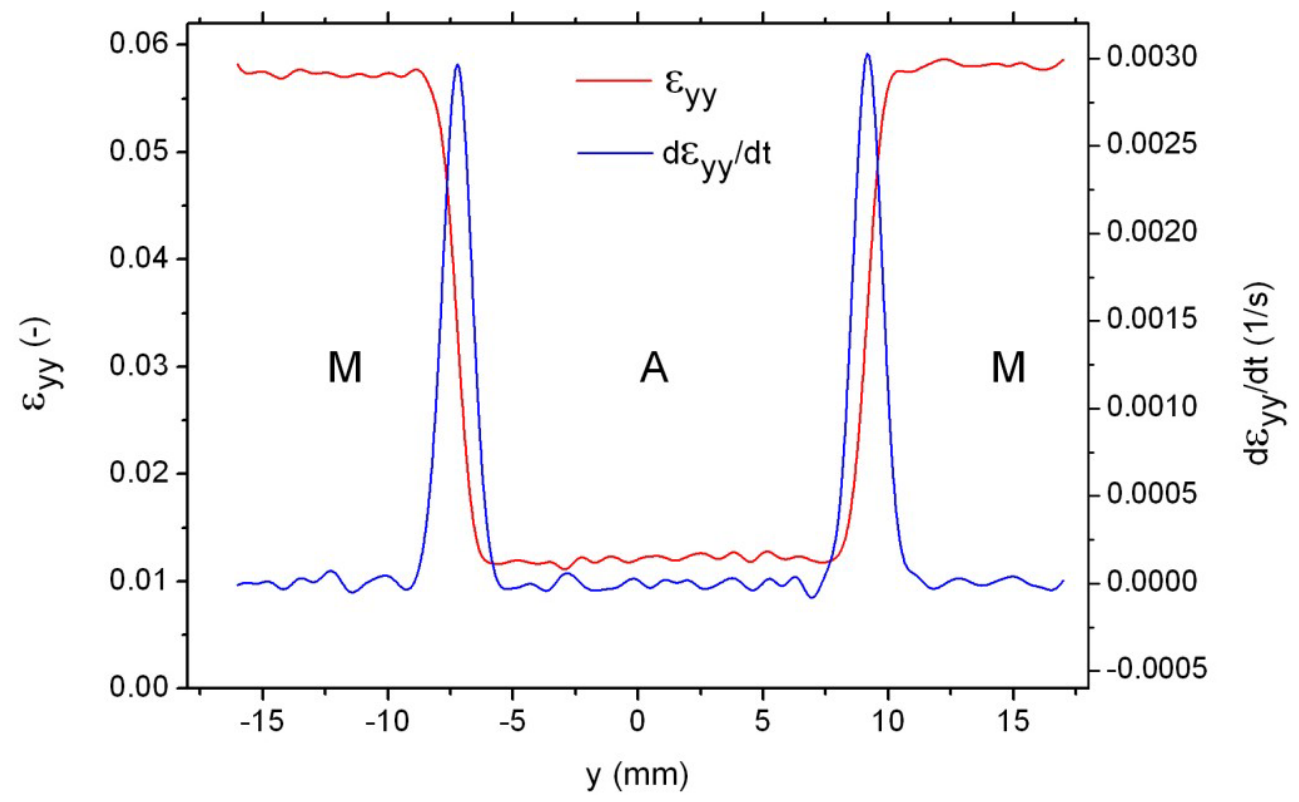

Fig. 3. Distribution of tensile strains and strain rates along the centerline of the specimen (macroscopic strain $\varepsilon_{y y}=3 \%$ ). $M$ and $\mathrm{A}$ indicate martensitic and austenitic regions, respectively. During localized deformation, additional transformation only occurs inside the narrow transition regions. 
For a more detailed analysis, local strains and strain rates along the specimen's centerline for one selected time step $\left(\varepsilon_{\mathrm{yy}}=3 \%\right)$ during localized deformation are presented in Fig. 3. These strain and strain rate data are representative for all macroscopic strains along the pseudoelastic plateau. At the selected time step, regions close to the grips have already transformed to martensite whereas the middle of the specimen is still austenitic. The tensile strains vary step-like but smoothly across the two phase interfaces. Fig. 2 and Fig. 3 highlight the inhomogeneous nature of strains and strain rates during localized deformation of pseudoelastic NiTi. Inside the martensitic bands, the strain values correspond to the end of the macroscopic stress-strain plateau (Fig. 1), whereas the strain inside the austenitic region corresponds to the onset of the stress-plateau. Fig. 3 also demonstrates that the ongoing deformation is limited to the interface regions: Tensile strain rates inside the fully transformed regions, as well as in the remaining austenitic region in the middle of the specimen, are equal to zero, and thus all deformation/ transformation must take place in the narrow transition regions in-between in order to provide the macroscopic elongation of the specimen enforced by the experimental procedure.

As the macroscopic deformation associated with the pseudoelastic loading and unloading plateaus is enforced at a constant macroscopic displacement rate, the phase interfaces also need to propagate along the gauge length at a constant velocity. Shaw and Kyriakides [1] suggested a simple functional relationship between the number of phase interfaces $\mathrm{n}$, the average interface velocity $v$, the macroscopic displacement rate $\dot{\delta}$, and the uniaxial transformation strain $\varepsilon_{t}$ :

$$
\dot{\delta}=\imath \cdot v \cdot \varepsilon_{t}
$$

From the temporal evolution of additional strain and strain rate data like the data sets presented in Fig. 3 (not shown here), an average interface velocity of $v=0.089 \mathrm{~mm} / \mathrm{s}$ can be determined. This velocity exactly matches the prediction for $v$ based on equation (1) with $\mathrm{n}=2$ (see Fig. 2 and Fig. 3 ), $\dot{\delta}=0.0083 \mathrm{~mm} / \mathrm{s}$, and $\varepsilon_{t}=4.7 \%$ (see Fig. 1). Therefore, despite the finite width and the inclination of the macroscopic phase interfaces in our flat ribbon specimens, and even though strains and strain rates exhibit rather complex distributions at the interfaces (see also Fig. 4), our experimental results confirm that the simple relationship (equation (1)) originally proposed by Shaw and Kyriakides represents a remarkably accurate approximation of local interface velocities.

One additional important experimental result is the pronounced difference between maximum local strain rates in the center of the interface and the macroscopic strain rate (calculated by dividing the crosshead displacement rate by the free length of the specimen): At $29.67 \times 10^{-4} 1 / \mathrm{s}$ (see Fig. 3), the former is one magnitude higher than the latter $\left(\dot{\delta} / 1_{0}=2.38 \times 10^{-4} 1 / \mathrm{s}\right)$. As all deformation takes place in the narrow transition region between martensitic and austenitic bands, the local rate of deformation clearly affects the local, exothermic release of heat due to the martensitic transformation [13]. Obviously, macroscopic strain rates are not well suited to characterize the thermo-mechanical loading conditions during localized deformation of pseudoelastic NiTi because they depend by definition on $1_{0}$, whereas the interface velocity does not (see equation (1)). In contrast, the simple experimental parameter $\dot{\delta}$ is directly related to the interface velocity $v$. Crosshead displacement rates are therefore better suited for the evaluation and comparison of experimental data because they provide information on both the testing procedure and on the local, thermo-mechanical loading conditions.

Focusing on one austenite-martensite interface, Fig. 4a) shows a more detailed strain map at increased resolution of the transition region between martensite and austenite $\left(\varepsilon_{\mathrm{yy}}=3 \%\right)$. Local strain rates along the three parallel lines, labeled left, center, and right in Fig. 4a), are shown in Fig. 4b). The phase interface can only be described as a straight boundary with a constant angle of inclination when iso-strain contours with strains near 3$4 \%$ are considered as characteristic for the interface shape and orientation. Indeed, iso-strain contours with strains near either the onset or the end of the pseudoelastic plateau approach the free edges of the specimen almost perpendicularly. Moreover, the close-up of the strain distribution highlights that the geometry of the interface varies across the width of the specimen; the transition region between austenite and martensite broadens towards the free edges of the specimen. In consequence, local strains near the specimen edges increase somewhat slower when the interface propagates at constant velocity. This finding is reflected in the strain rate data (Fig. 4b)), where the maximum strain rates of the left and right data sets are smaller than the one associated with the centerline. Moreover, the centerline plot is highly symmetric, whereas the left line plot bulges out on the right and, similarly, the right line plot broadens on the left side. These observations are a general feature of the interface (i.e., similar strain distributions and strain rate data are observed at all time steps corresponding to localized deformation along the pseudoelastic plateau). Finally, comparing Fig. 2-Fig. 4, one can estimate the finite width of the phase interfaces in the ribbon material used here as $\sim 4-5 \mathrm{~mm}$. The size of the regions across which the interfaces are spread is therefore of a similar order of magnitude as the specimen width. This observation confirms theoretical predictions for thin tensile specimens [14].

In closing, we note that the first experimental results presented here demonstrate that the DIC technique is a promising approach to study even the subtle details of strain states and strain rates near propagating interfaces. While we have only considered the most dominant (tensile) strain, the DIC data do in principle also allow for a 
complete evaluation of transverse and shear strains. Further work is required to fully characterize the complex strain states near the macroscopic phase interfaces in pseudoelastic NiTi. Such analyses are currently underway.

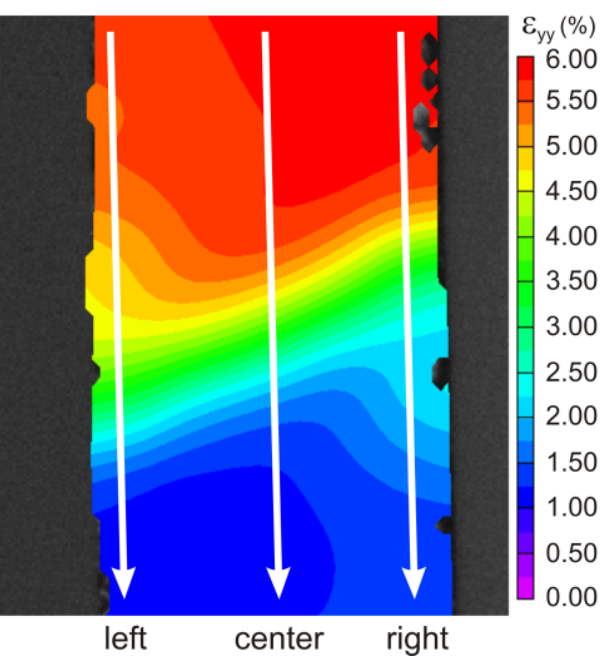

a)

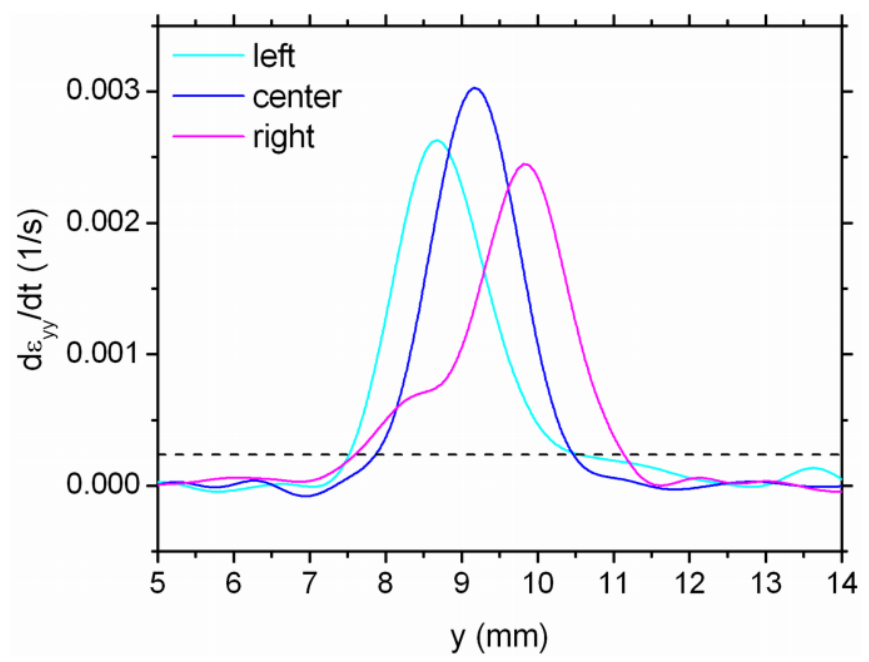

b)

Fig. 4. a) Distribution of tensile strains across a martensite-austenite interface. The transition region broadens towards the edges of the specimen. b) Strain rates along the three parallel lines shown in a). The dashed line represents the macroscopic strain rate of $2.38 \times 10^{-4} 1 / \mathrm{s}$, which is one magnitude below the maximum local strain rates.

\section{Summary and Conclusions}

We have used digital image correlation (DIC) to investigate the local strains and strain rates in a pseudoelastic NiTi ribbon subjected to a displacement controlled uniaxial tensile test. The distribution of tensile strains can be determined with high spatial resolution and clearly highlights the inhomogeneous nature of transformation/ deformation during pseudoelastic straining of thin NiTi specimens: Only the material in the phase interface itself contributes to the elongation of the specimen during straining along the pseudoelastic plateaus (both during the forward and reverse transformation), whereas strain rates are zero inside the fully austenitic or martensitic regions in the gauge length. The distribution of local strain rates along the gauge length confirm that the macroscopic phase interfaces propagate along the gauge length at constant velocity when a constant crosshead displacement rate is applied during testing. The maximum local strain rates are one order of magnitude higher than the macroscopic strain rate, which is frequently used to characterize mechanical testing procedures. Moreover, we have performed a careful analysis of tensile strains and strain rates in the close vicinity of a propagating phase interface. Interestingly, the width of the interfaces $(\sim 4-5 \mathrm{~mm})$ increases towards the free edges of the ribbon specimen, and iso-strain contours approach these edges at varying angles. In the light of these more detailed data on the strain distribution near the phase interfaces, the definition of a single angle of interface inclination appears to be a strong simplification. Further DIC analyses, including a closer analysis of transverse and shear strains, may provide a pathway towards a more detailed understanding of phase boundary properties and dynamics during localized deformation of NiTi.

\section{References}

[1] J. A. Shaw and S. Kyriakides, J. Mech. Phys. Solids 43, 1243 (1995)

[2] J. A. Shaw and S. Kyriakides, Acta Mater. 45, 683 (1997)

[3] J. A. Shaw and S. Kyriakides, Int. J. Plast 13, 837 (1998)

[4] Y. Liu, Y. Liu and J. V. Humbeeck, Scr. Mater. 39, 1047 (1998)

[5] P. Feng and Q. P. Sun, J. Mech. Phys. Solids 54, 1568 (2006)

[6] P. H. Leo, T. W. Shield and O. P. Bruno, Acta Metall. Mater. 41, 2477 (1993)

[7] S. Daly, G. Ravichandran and K. Bhattacharya, Acta Mater. 55, 3593 (2007)

[8] C. Efstathiou and H. Sehitoglu, Scr. Mater. 59, 1263 (2008) 
[9] J. Olbricht, A. Schäfer, M. F. X. Wagner and G. Eggeler, "Characterization of transformation localization during pseudoelastic cycling of NiTi", International Conference on Shape Memory and Superelastic Technologies SMST, Tsukuba, Japan 2007, edited by S. Miyazaki (ASM International), p. 47

[10] A. Schäfer, J. Olbricht and M. F. X. Wagner, "Monitoring localized deformation of pseudoelastic NiTi subjected to uniaxial loading", International Conference on Martensitic Tranformations ICOMAT SantaFe, USA 2008, in print

[11] R. Heinen, K. Hackl, W. Windl and M. F.-X. Wagner, Acta Mater. 57, 3856 (2009)

[12] M. F. X. Wagner and W. Windl, Acta Mater. 56, 6232 (2008)

[13] M. A. Iadicola and J. A. Shaw, J. Intell. Mater. Syst. Struct. 13, 157 (2002)

[14] Q.-P. Sun and Z. Zhong, Int. J. Plast 16, 1169 (2000) 